\title{
Numerical Study of the Composite Effects of Uneven Street Canyons and Time-varying Inflows on the Air Flows and Pollutant Dispersion
}

\author{
Weijun Li ${ }^{1}$, Yuanping He ${ }^{1,2}$, Yunwei Zhang ${ }^{1}$, Qingxiang Shui ${ }^{1}$, Xinyi Wu ${ }^{1}$, Chuck Wah. Yu ${ }^{1,3}$, \\ Zhaolin $\mathbf{G u}^{1^{*}}$ \\ ${ }^{1}$ Department of Earth and Environmental Sciences, Xi'an Jiaotong University, Xi'an 710049, China \\ ${ }^{2}$ Department of Architecture and Civil Engineering, City University of Hong Kong, Kowloon Tong, HKSAR, Hong Kong \\ ${ }^{3}$ International Society of the Built Environment (ISBE), Milton Keynes, MK78HQ, UK
}

\begin{abstract}
The dispersion behavior of pollutants in realistic street canyons is dominated by the pattern of airflow, which is closely linked to the building layout and air inflow at the street canyon boundary. Although uneven building layouts and timevarying inflows have been individually investigated for their influence on air flow pattern and pollutant dispersion in street canyons, the combined effects of these two factors have not been examined. In this study, four street canyon models, one even and three uneven models with different occupying ratios of high-rise buildings, were numerically studied using largeeddy simulation under steady inflow and time-varying inflow conditions. The results showed that both the uneven building layouts and the time-varying inflow condition enhanced the air flow strength and pollutant removal efficiency inside the street canyon. However, the composite effects of these factors were not simply additive. The worst pollutant removal efficiency was observed for air flow in the even street canyon with the steady inflow, which is the typical benchmark case in studies. With the time-varying inflow, the induced updrafts prevented the relatively tall high-rise buildings from restricting the height of the pollutant dispersion during the inflow in the valley. The pattern of the pollutant isoconcentration plane and the time-series curves of normalized concentrations in the even and uneven street canyon models exhibited fluctuant features with the time-varying inflow.
\end{abstract}

Keywords: Airflow pattern; Large-eddy simulation; Pollutant dispersion; Time-varying inflow; Uneven building layouts.

\section{INTRODUCTION}

Air pollution in urban areas is a major issue caused by the increase of road transportation and ongoing urbanization in China (Chan and Yao, 2008). High pollutant concentrations in the lower atmosphere do harm to pedestrian health. In addition, outdoor air pollutants are able to penetrate indoors through ventilation systems, windows or building cracks, etc. Therefore, investigating the flow pattern and dispersion of pollutants in urban areas is critical. As the basic geometric units of urban areas, street canyon constitute the most common research object (Eliasson et al., 2006; Fazia and Helmut, 2006; Zhang et al., 2011; Krecl et al., 2015; Wang et al., 2015). Numerous investigations have been undertaken to study the airflow and pollutant dispersion in street canyons, including laboratory-scale experiments (Baik et al., 2000; Ahmad et al., 2005; Scungio et al., 2013; Zhang et al., 2013),

\footnotetext{
* Corresponding author.

Tel.: +86-1-308-896-0683; Fax: +86-8-339-5100

E-mail address: guzhaoln@mail.xjtu.edu.cn
}

field measurements (Li et al., 2007; Kumar et al., 2009), and computational fluid dynamics (CFD) simulations (Sabatino et al., 2007; Li et al., 2009; Zhang et al., 2012).

Pollutant dispersion behavior is dominated by the flow pattern in street canyons, which is closely linked to the building layout and the air inflows at street canyon boundary. Flow patterns with steady and perpendicular approaching inflows in even ideal street canyons can be categorized into four types (Oke, 1988; Vardoulakis et al., 2003; Li et al., 2006; Xie et al., 2006; Li et al., 2009; Zhang et al., 2019), i.e., isolated roughness flow (aspect ratio [AR] $<0.3)$, wake interference flow $(0.3<\mathrm{AR}<0.7)$, skimming flow $(0.7<\mathrm{AR}<1.57)$ and multi-vertex flow (AR > 1.57). However, actual street canyons always present an uneven layout, signifying that buildings on one side of a street have different heights from those on the other side. Hang et al. (2012) demonstrated building height variations strengthen street pollutant dilution in front of taller buildings but reduce that in streets behind taller buildings. Lin et al. (2016) reported that the upstream taller buildings lead to a much weaker counter-clockwise vortex within the target $2 \mathrm{D}$ street canyon. Zhang et al. (2019) also found the downstream taller building weakened pollutant dispersion capacity in $2 \mathrm{D}$ 
street canyon models. Above mentioned research results were based on the uneven street canyons with unilateral buildings of the same height. Gu et al. (2011) firstly identified the effects of building layout on the airflow pattern in uneven street canyons with step-up or step-down notches. They simulated uneven street canyons by changing the occupying ratio of high buildings (ORHB) in the computational domain along the street and their bilateral allocation as well as the combinations of step-up and step-down notches. Specifically, they performed numerical simulations for four cases that involved different ORHB values $(0,0.25,0.50$ and 0.75$)$, and the simulation results demonstrated the presence of tilting, horizontal divergence and convergence of the wind streamline in the uneven street canyons; moreover, the results revealed that pollutant removal efficiencies in the uneven street canyons were much higher than those in even ones. Similarly, Nelson et al. (2007) conducted field measurements in the Park Avenue street canyon (Oklahoma City, Oklahoma, USA), and the measurement results revealed wind downdraft and horizontal divergence resulting from an uneven building layout. Wind tunnel experiments performed by Sagrado et al. (2002) on even and uneven street canyon models indicated that the recirculating region was broken; this recirculation resulted in a decrease in the pollutant concentration inside the uneven street canyon model $(\mathrm{ORHB}=0$, but the bilateral buildings have different heights) compared with the even one.

Under the consideration of steady air inflows at the boundary, in situ measurements and CFD simulations have demonstrated the formation of a recirculating vortex inside an even street canyon and a strong shear layer at the roof level when the inflow direction was perpendicular to the street (Oke, 1998; Vardoulakis et al., 2003; Li et al., 2008) this scenario has the worst effect on pollutant transport in street canyons. The assumption of the boundary air inflows at a perpendicular incidence to the street is usually adopted for numerical simulations of airflow and pollutant dispersion in street canyons, irrespective of the wind direction of actual time-varying inflows; this study focused on the wind velocity of time-varying inflows. Several studies have demonstrated that a time-varying inflow could affect the variation in the downstream airflow field and mass transport in the atmospheric boundary layer (Liu et al., 2002; Zeng et al., 2007; Zhang et al., 2011; Duan et al., 2018; Zhang et al., 2018). After the passage of a cold front, regular gusty wind disturbances are observed as a typical time-varying inflow. Gusty wind disturbances have been regarded as an efficient driving force of the vertical transport of momentum and mass. By updating the velocity condition of the lateral boundary every $15 \mathrm{~min}$ in a simulation, Liu et al. (2002) showed that introducing the time-varying inflows into an urban wind field could significantly enhance the dispersion of pollutants. Zhang et al. (2011) identified an obvious intermittent feature in a street canyon and flapping of the shear layer near the roof layer under time-varying inflows; they found that these could significantly influence pollutant dispersion efficiency. Duan et al. (2018) observed a small but statistically significant response to inflow perturbations in the turbulent flow inside an ideal street canyon after incorporating time-periodic perturbations into the streamwise velocity component.

As mentioned above, the individual effects of uneven building layouts or time-varying inflows on the airflow pattern and pollutant dispersion in street canyons have already been investigated separately; however, the composite effects of uneven building layouts and time-varying inflows on the airflow pattern and pollutant dispersion in street canyons remain unexplained. The present study established four street canyon models (one even model and three uneven models with different ORHBs of $0.25,0.50$ and 0.75 ) for numerical simulations under time-varying boundary inflows; these models are the same as those used in the previous study by the authors' research group to examine the steady boundary inflow (Gu et al., 2011). The time-varying boundary air inflow was acquired by preprocessing recorded highfrequency wind velocity data measured at the Qujiang campus of Xi'an Jiaotong University in Xi'an, China. Subsequently, the instantaneous and statistical airflow structures and pollutant dispersion processes inside and above the simulated street canyons were investigated to demonstrate the composite effects of uneven building layout and time-varying boundary air inflows.

\section{STREET CANYON MODELS AND MESHING}

By referring to the previous study conducted by the authors' research group (Gu et al., 2011), the present study established four street canyon models, as illustrated in Fig. 1, with Model 1 denoting an even street canyon and Models 24 denoting uneven street canyons containing step-up and step-down parts. The step-up and step-down notches were considered to be separated in Models 2 and 4 but adjoined in Model 3. The Cartesian coordinate system was adopted for the models, with the $x$-axis representing the air inflow direction, the $y$-axis representing the axial direction of the street canyon models, and the $z$-axis representing the height direction of the buildings.

The computational grid is a crucial element in CFD because it determines the level of resolution of a flow field and the simulation accuracy. In the computational domain in this study, both the surfaces of the buildings and the ground were determined to be a solid wall without slip. The surrounding grids must be refined. Theoretically, in the numerical model using a wall function, the condition $z^{+}=$ $\left(\Delta z u \_\tau\right) / v \leq 100$ should be satisfied (Pope, 2000), where $\Delta z$ is the height of the first layer of grids near the wall, $u_{\tau}$ is the shear velocity, and $v$ is the kinematic viscosity coefficient. When the free stream velocity is $3.5 \mathrm{~m} \mathrm{~s}^{-1}$ (also serving as the maximum velocity of the time-varying inflows adopted in this study) and the height of computational domain is $45 \mathrm{~m}, \Delta z$ should not be greater than $0.02 \mathrm{~m}$, as estimated by $z^{+}$. Therefore, the height of the first layer grid near the wall was set to $0.02 \mathrm{~m}$, and the remaining grids gradually increased perpendicular to the wall surface with the expansion ratio of 1.2. The total cell number for all models ranged from 1.6 to 2.2 million. As an example, the grids of Model 2 are depicted in Fig. 2. 


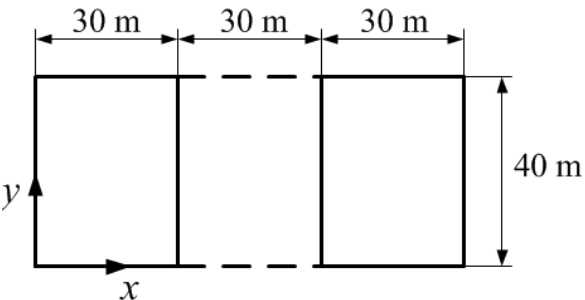

(a)

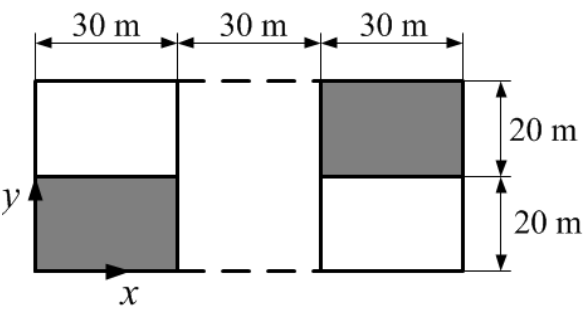

(c)

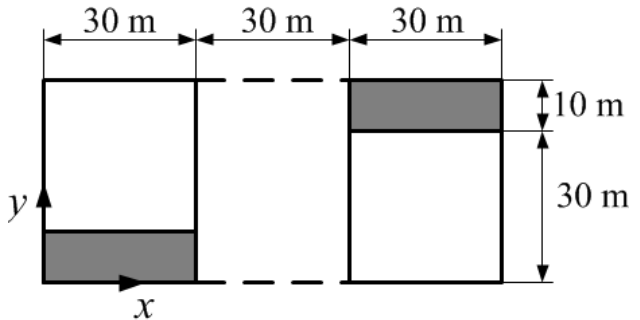

(b)

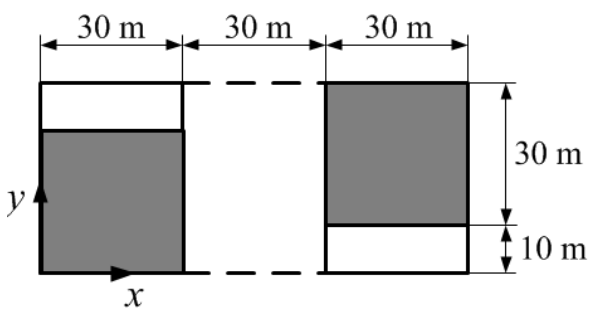

(d)

Fig. 1. Planar graphs of (a) Model 1, (b) Model 2, (c) Model 3, and (d) Model 4 in the horizontal $x-y$ plane. Solid-lined squares represent relatively low buildings with heights of $30 \mathrm{~m}$, and solid-lined gray squares represent relatively tall buildings with heights of $45 \mathrm{~m}$. The computational domain in the vertical direction, $z$, is 2.5 times the height of the low buildings.

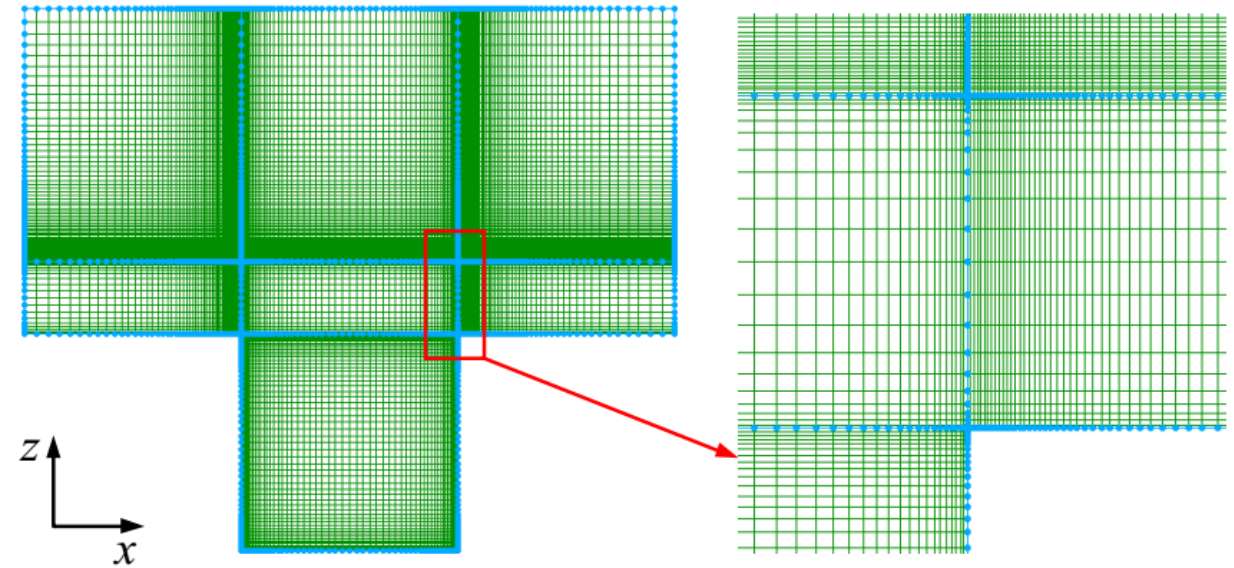

(a)

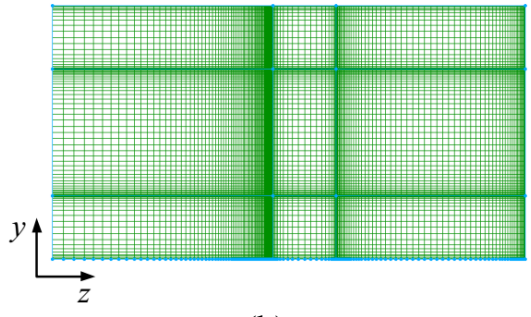

(b)

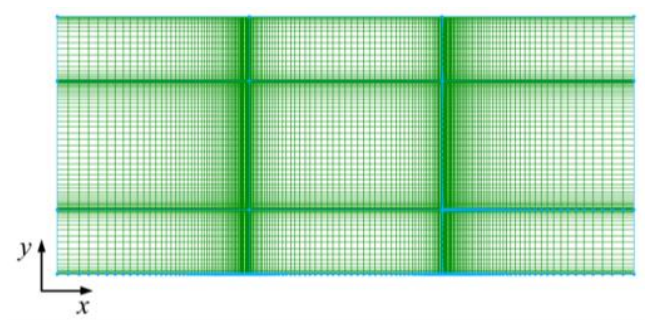

(c)

Fig. 2. Grids on the (a) $x-z$ plane, (b) $y-z$ plane, (c) $x-y$ plane for Model 2.

\section{METHODOLOGY}

\section{Large-eddy Simulation Equations}

Tominaga and Stathopoulos (2011) investigated the performance of Large-eddy Simulation (LES) and Reynolds Average Navier-Stokes (RANS) equations in modeling pollutant dispersion in a street canyon. The numerical results indicated that LES modeling yielded superior results to the conventional RANS equations with respect to the modeling of the distribution of the mean concentration of pollutants. Moreover, compared with LES simulation, RANS simulation underestimated the turbulence diffusion in the street canyon. Therefore, LES was adopted for calculating turbulent flows in the various street canyon models in this study. In LES, only large-scale fluctuations are directly calculated by solving the Navier-Stokes equations, and small-scale fluctuations 
are filtered out and evaluated using a certain subgrid stress model. Therefore, the governing equations for LES can be expressed as follows:

$$
\begin{aligned}
& \frac{\partial \bar{u}_{i}}{\partial \bar{x}_{i}}=0 \\
& \frac{\partial \bar{u}_{i}}{\partial t}+\frac{\partial \bar{u}_{i} \bar{u}_{j}}{\partial x_{j}}=-\frac{1}{\rho} \frac{\partial \bar{p}}{\partial x_{i}}+v \frac{\partial^{2} \bar{u}_{i}}{\partial x_{j} x_{j}}+\frac{\partial \bar{\tau}_{i j}}{\partial x_{j}}
\end{aligned}
$$

where $\overline{u_{i}}, \overline{u_{j}}$ are the resolved-scale velocities in the $i$ and $j$ directions, respectively; $\bar{p}$ is the resolved-scale pressure; and $\overline{\tau_{i j}}$ is subgrid stress, which can be parameterized as follows:

$$
\bar{\tau}_{i j}=2 v_{S G S} \bar{S}_{i j}+\frac{1}{3} \delta_{i j} \tau_{k k}
$$

where $v_{S G C}$ is the subgrid viscosity coefficient and $\bar{S}_{i j}$ is the resolved strain-rate tensor. In this paper, $\overline{\tau_{i j}}$ was calculated using Smagorinsky eddy-viscosity model (Smagorinsky, 1963). Thus, $v_{S G C}$ can be expressed as follows:

$v_{S G S}=\left(C_{s} \Delta\right)^{2}\left(\bar{S}_{i j} \bar{S}_{i j}\right)^{1 / 2}$

where $C_{s}$ is the Smagorinsky constant with a suggested value of 0.18 , and $C_{s} \Delta$ is equivalent to mixed length.

Pollutant concentration is represented by the scalar $C$. The transport equation of scalar $C$ is as follows:

$$
\frac{\partial \bar{C}}{\partial t}+\bar{u}_{j} \frac{\partial \bar{C}}{\partial x_{j}}=K_{C} \frac{\partial^{2} \bar{C}}{\partial x_{j} x_{j}}+\frac{\partial}{\partial x_{j}}\left(\bar{u}_{j} \bar{C}_{i}-\overline{u_{j} C}\right)+S_{c}
$$

where $\overline{u_{j}}$ is the time-averaged velocity components, $K_{c}=$ $v_{t} / S_{c t}$ is the turbulent eddy diffusivity of pollutants, $v_{t}$ is the kinematic eddy viscosity, and $S_{c t}$ is the turbulent Schmidt number representing the ratio of momentum diffusivity and mass diffusivity. In CFD simulations, $S_{c t}$ is usually treated as a constant ranging from 0.2 to 1.3 (Kim and Baik, 2004; Bady et al., 2008; Hang et al., 2009; Yim et al., 2009); in the present study, $S_{c t}$ was set to $1 . S_{c}$ is the emission source intensity. For a situation in which vehicles traverse the street canyon, two volume sources ( $x$ : 34-36 $\mathrm{m}$ and 54-56 m; $y$ : $0-40 \mathrm{~m} ; z: 0-1 \mathrm{~m}$ ) were set in the computational domain with an emission rate of $10 \mu^{-3} \mathrm{~s}^{-1}$, as presented in Fig. 3.

\section{Boundary Conditions}

The free-stream wind profile above an urban roughness layer exhibits an exponential distribution (Baik and Kim, 1999):

$u(z)=U_{r e f}\left(\frac{z-H_{1}}{H-H_{1}}\right)^{0.299}$

where $U_{\text {ref }}$ is the reference wind velocity determined by the wind speed at the top of the computational domain, $H_{1}$ is the

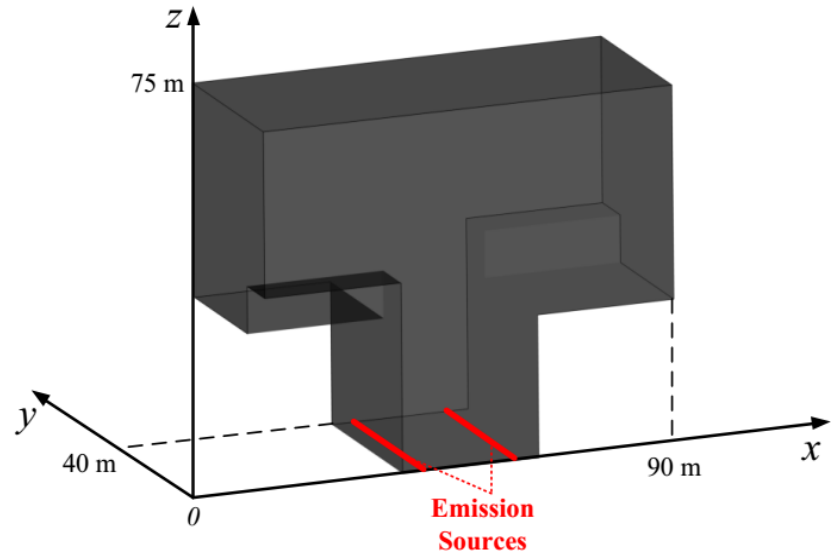

Fig. 3. Sketch map of emission sources for Model 2.

height of relatively low buildings ( $30 \mathrm{~m}$ in this study), and $H$ is the height of computational domain (75 $\mathrm{m}$ in this study).

To acquire time-varying inflows, this study obtained a time-series of wind velocity data at the Qujiang campus of $\mathrm{Xi}$ 'an Jiaotong University in Xi' an, China, by using a threedimensional ultrasonic anemometer with a sampling frequency of $20 \mathrm{~Hz}$. The three-dimensional ultrasonic anemometer has a wind speed measurement range of $0-65 \mathrm{~m} \mathrm{~s}^{-1}$, with a minimum resolution of $0.01 \mathrm{~m} \mathrm{~s}^{-1}$ and a measurement precision of $\pm 1.5 \%$. The data recorded on December 13, 2018, at 14:00-15:00 LT (local time $=\mathrm{UTC}+12 \mathrm{~h}$ ) are illustrated in Fig. 4(a).

It was unrealistic to directly use the measured highfrequency wind velocity data as the time-varying inflows in our numerical simulations because of the considerably large amount of data. To obtain low-frequency time-series data as the time-varying inlet velocity condition, this study derived the average value of wind velocities over a certain period. In such an averaging process, the average time is a crucial parameter that determines the quality of acquired low-frequency inflow. With respect to the power spectrum, the area under the premultiplied power spectrum $f \Phi_{u u}$ is proportional to the turbulent kinetic energy (TKE) contribution in a specific frequency range (Kim and Adrian, 1998; Smits et al., 2011; Wang and Zheng, 2016). To retain the component with the maximum TKE, the average time should be smaller than the corresponding value of the peak point in the premultiplied power spectrum. Notably, turbulent fluctuation constitutes the object of the premultiplied power spectrum. Therefore, a detrending process must be performed to strip the interference of non-turbulence-related large-scale trends (Hutchins et al., 2012; Li et al., 2019). The detrended turbulent fluctuation is shown in Fig. 4(b).

On the basis of the detrended turbulent fluctuation, the power spectrum density function $\phi_{u и}$ was estimated using Welch's average periodogram method (Welch, 1967) with a Hanning window and overlapped segment (10\% of the window length); the premultiplied power spectrum with frequency is shown in Fig. 5. A strengthening peak was observed at approximately $f=0.02 \mathrm{~Hz}$ (or $T=1 / f=50 \mathrm{~s}$ ) in the premultiplied power spectrum. Therefore, the raw velocity data were smoothed with an average time of $50 \mathrm{~s}$ to obtain a 


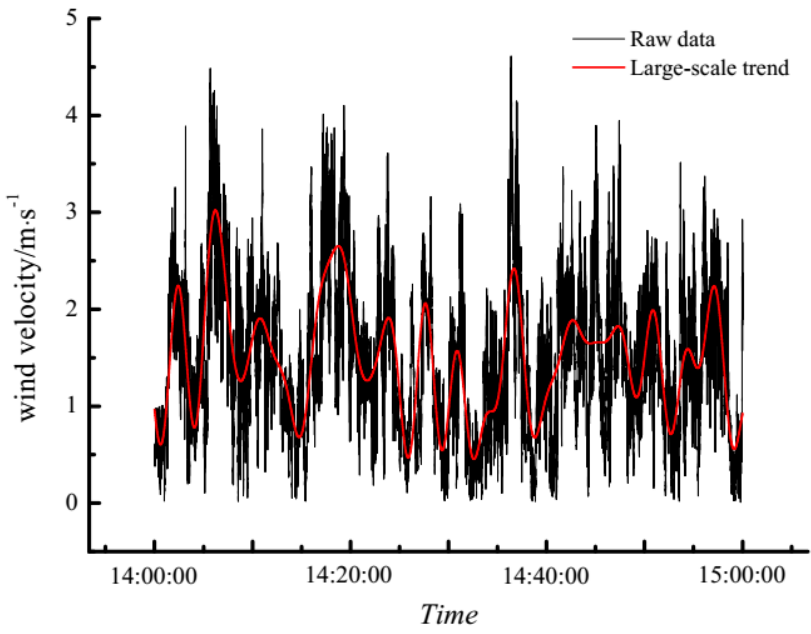

(a)

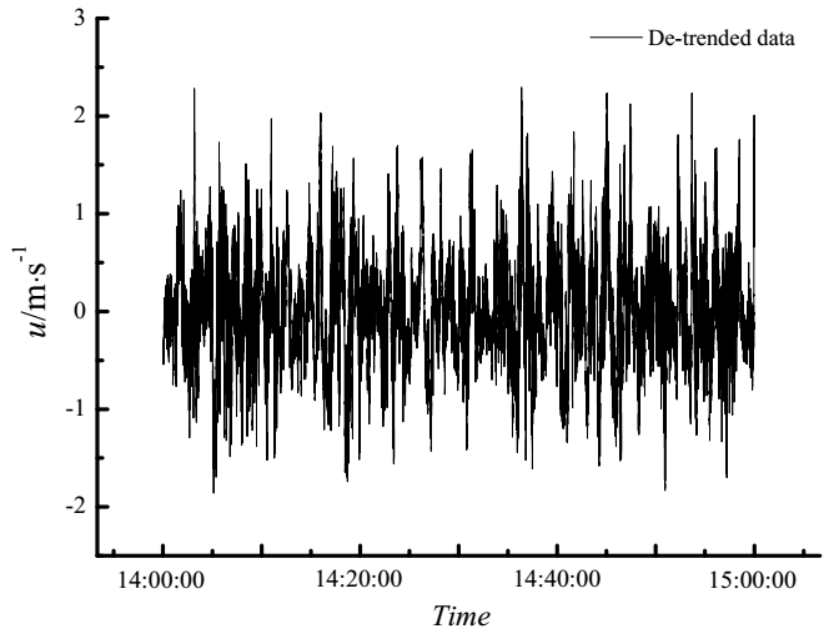

(b)

Fig. 4. Field-measured wind velocity data: (a) raw data and large-scale trend, (b) detrended turbulent fluctuation.

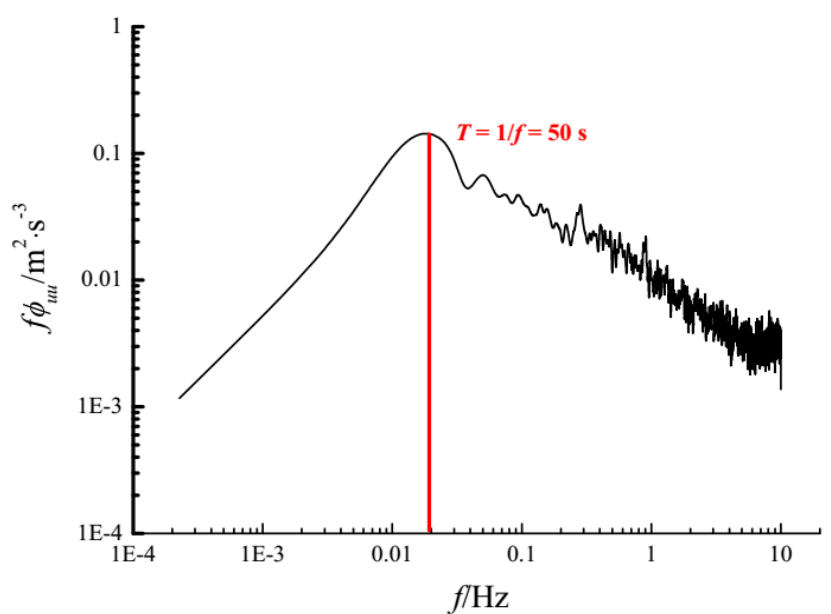

Fig. 5. Premultiplied power spectrum with frequency.

set of low-frequency wind velocity data, which served as the reference wind velocity (the intermediate values were acquired by linear interpolation), as presented in Fig. 6. According to Eq. (6), a time-varying boundary velocity condition was formulated and applied to the inlet boundary. For Models 1, 2, 3 and 4, the cases under the time-varying inflow condition were named Cases 1, 2, 3, and 4, respectively. For comparison, a steady inflow condition with a reference velocity of $1.5 \mathrm{~m} \mathrm{~s}^{-1}$ was also applied to Models 1, 2, 3 and 4, with the corresponding cases being named Case $1 s, 2 s, 3 s$, and $4 s$, respectively. Notably, the wind direction was always perpendicular to the inlet boundary in both the steady and time-varying inflow conditions.

To clarify the reasonableness of the average time of $50 \mathrm{~s}$ evaluated by the premultiplied power spectrum with frequency, evaluated by the premultiplied power spectrum with frequency, four additional average times of 12, 25, 100, and $200 \mathrm{~s}$ were adopted to smooth the measured high-frequency wind velocity data, forming four sets of time-varying inflows. In particular, the average time of $200 \mathrm{~s}$ is close to the period of regular gusty wind disturbances that are always found in the atmospheric boundary layer. For Model 2 in this study, pollutant dispersion was simulated under five sets of time-varying inflows with different average times, and the comparison of the simulated results is provided in Appendix A.

Flow field was fully developed at the outlet; thus, a zerogradient boundary condition was adopted. A symmetry boundary condition was used at the boundaries in the $y$ direction. The top boundary was specified as a slip condition with zero vertical variation, and nonslip wall condition was applied to all solid walls. In addition, the inlet turbulence kinetic energy was generated by extracting the turbulence kinetic energy on the $y-z$ plane at $x=45 \mathrm{~m}$. A particular turbulent viscosity wall function-nutkWallFunction in Open Field Operation and Manipulation (OpenFOAM) was applied to all solid walls. The nutkWallFunction wall function provides a turbulent viscosity condition based on the turbulent kinetic energy (Liu, 2016).

\section{Algorithm}

Because of actual time-varying inflow conditions and uneven building layouts, airflow in street canyons is not steady. During the process of solving unsteady flow problems, the discrete time term should be included in the NavierStokes equations. If the boundary conditions are constant, a traditional unsteady algorithm such as the Pressure-Implicit with Splitting of Operators (PISO) algorithm (Issa, 1986) can be used to derive the instantaneous flow field at a certain moment, which is only the convergent solution of all algebraic equations at the present moment (not the convergent solution of the entire field under the specific boundary condition at the present moment). When the boundary velocity condition varies with time, the propagation of nonconvergence error occurs. To solve the flow fields under time-varying inflow conditions more accurately, a new scheme based on the standard Semi-Implicit Method for Pressure- Linked Equations (SIMPLE) algorithm (Patankar and Spalding, 1976) was adopted in this study. The steps are described as follows:

I. Initialize the physical time $\left(t=t_{0}\right)$ and boundary condition $\left[u^{0}=f\left(t_{0}\right)\right]$. 


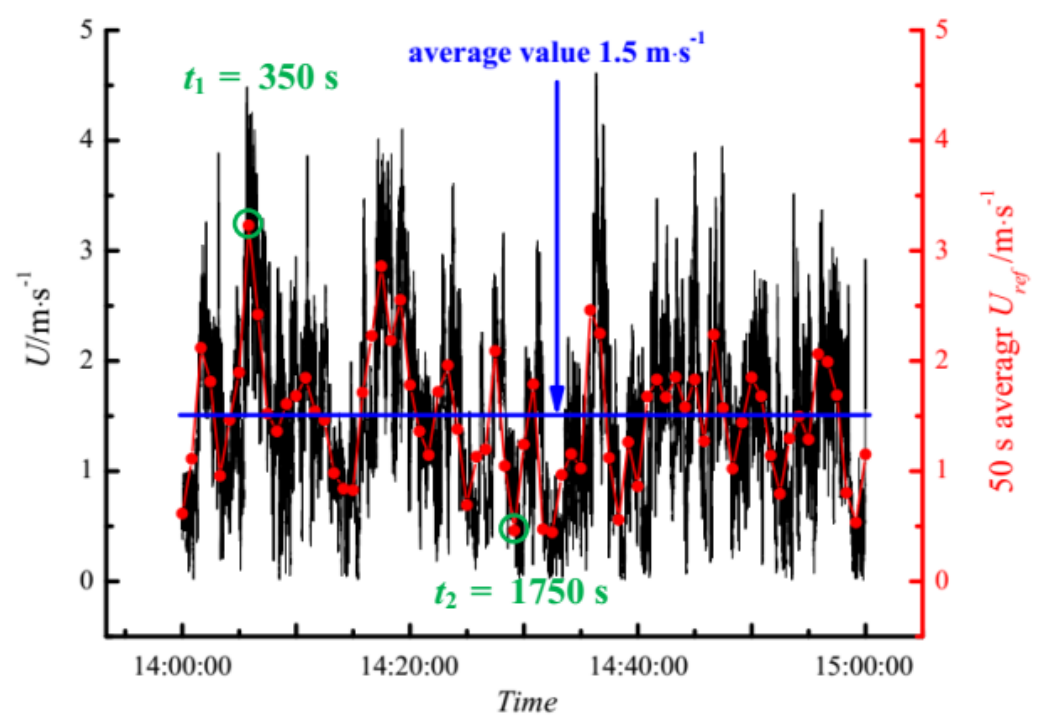

Fig. 6. Low-frequency wind velocities with an average value of $1.5 \mathrm{~m} \mathrm{~s}^{-1}$ were taken as the reference wind velocities.

II. Take the standard SIMPLE algorithm as the inner loop to acquire convergent field (For uneven models, there are no convergent fields, which are replaced by the average fields during a period). Notably, the scalar transport equation is not solved in the inner loop.

III. Initialize the physical time step $\Delta t$, and solve the scalar transport equation.

IV. Update velocity boundary condition $\left[u^{1}=f\left(t_{0}+\Delta t\right)\right]$.

V. Repeat Steps 2-4 as the outer loop.

This scheme transforms unsteady flow problems under time-varying inflow conditions into a series of intermediate processes with steady inflow. These intermediate processes are guaranteed to be convergent by controlling the external residual. Thus, the scheme can avoid the propagation of error (for more details of this scheme, please refer to the paper by Li et al., 2019). This scheme was formulated on the CFD platform of OpenFOAM (Su et al., 2019) to calculate the flow field and pollutant dispersion of the cases under time-varying inflow conditions. The number of inner iterative steps was set to 5000 , and the outer time step was $0.01 \mathrm{~s}$, which was estimated by Courant number in all simulations. The flow field and pollutant dispersion of the cases under steady inflow condition were calculated using the PISO algorithm. The time step was also set to $0.01 \mathrm{~s}$.

\section{RESULTS AND ANALYSES}

\section{Air Flow Patterns}

Air flow patterns are among the crucial elements in the analysis of the composite effects of uneven building layouts and time-varying inflow condition on pollutant dispersion in street canyons. In this study, seven sections were adopted to explain the three-dimensional flow fields in all cases, as shown in Fig. 7.

\section{Effects of Uneven Building Layouts}

The effects of uneven building layouts on the airflow patterns in the street canyon models were studied by comparing

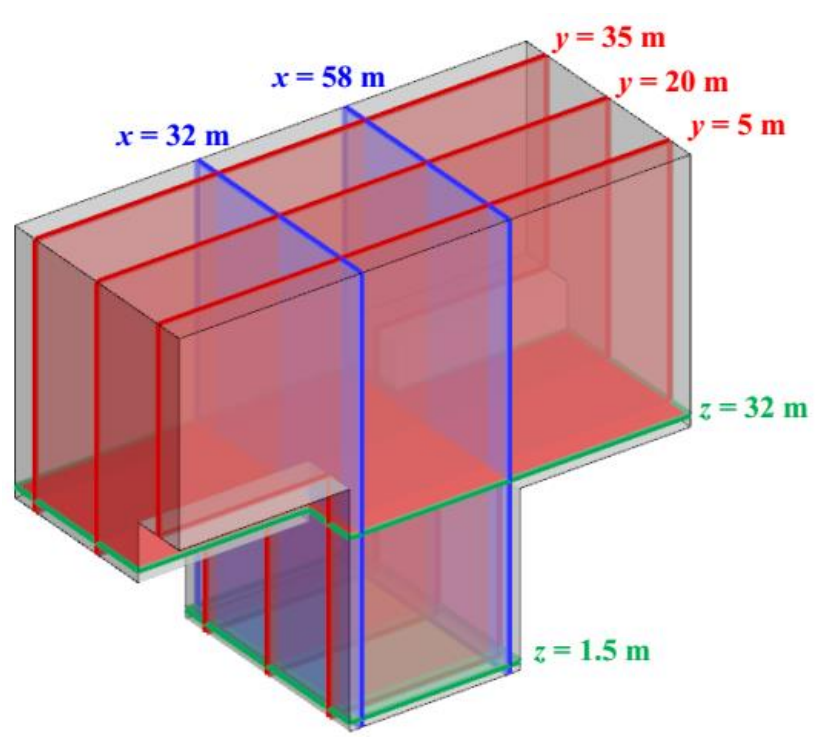

Fig. 7. Sketch map of sections, with Model 2 serving as an example.

the cases under a steady inflow condition (Case 1s, 2s, 3s and $4 \mathrm{~s}$ ). As the blank simulation, the velocity vector distributions of Case 1s in the sections (Fig. 7) are illustrated in Fig. 8 (velocity vectors were artificially magnified threefold to increase the readability of the illustrations). Because of the even building layout and cyclic boundary condition at the lateral boundaries in this case, the velocity vector distributions in all sections in the $x-z$ plane were similar; therefore, only the result obtained at $y=20 \mathrm{~m}$ is displayed. Consistent with results obtained in previous studies (Sabatino et al., 2007; Li et al., 2009), a recirculating primary vortex and several secondary vortices were formed inside the street canyon. Notably, the primary vortex was not completely perpendicular to the $y$ direction because of the influence of the secondary vortices.

The velocity vector distributions for Case $2 \mathrm{~s}$ are displayed 


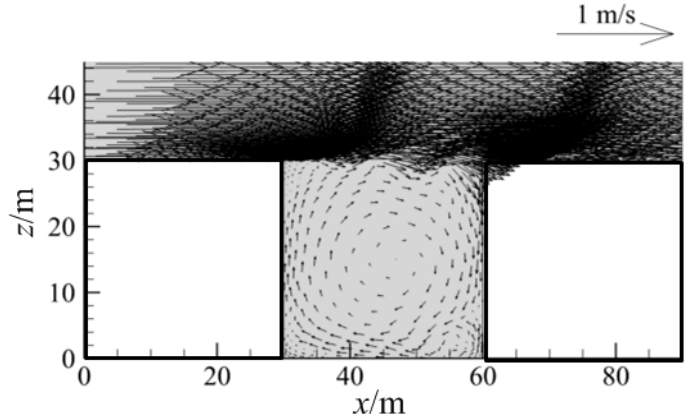

(a)

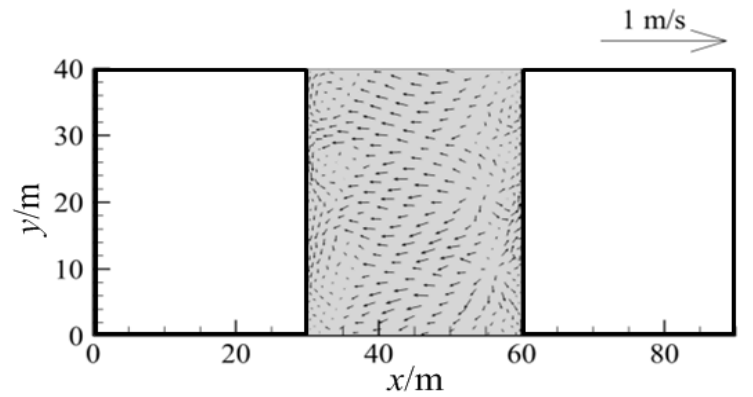

(d)

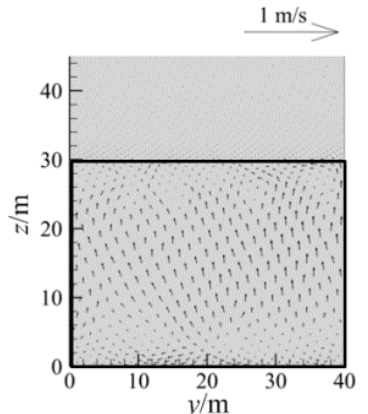

(b)

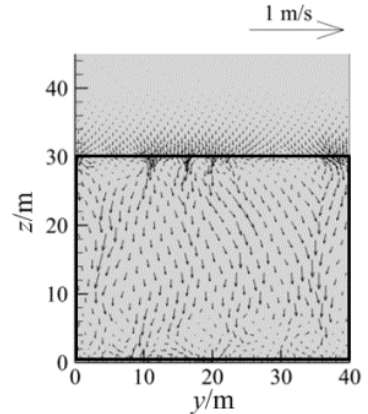

(c)

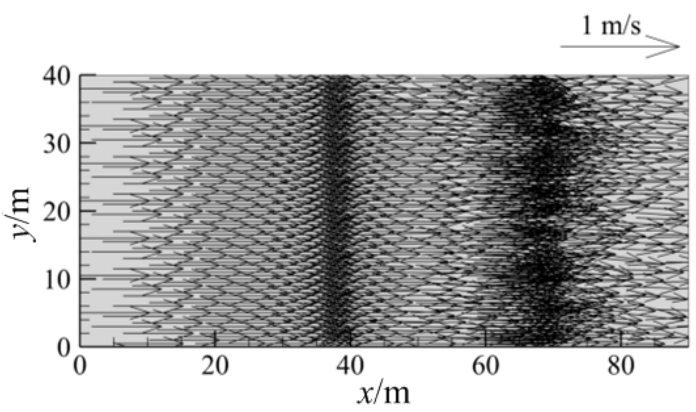

(e)

Fig. 8. Velocity vector distributions for Case $1 \mathrm{~s}$ in the (a) $x-z$ plane at $y=20 \mathrm{~m}$, (b) $y-z$ plane at $x=32 \mathrm{~m}(2 \mathrm{~m}$ away from leeward wall), (c) $y-z$ plane at $x=58 \mathrm{~m}$ (2 m away from windward wall), (d) $x-y$ plane at $z=1.5 \mathrm{~m}$ (pedestrian level), (e) $x-$ $y$ plane at $z=32 \mathrm{~m}$.

in Fig. 9. In contrast to those observed for Case 1s, the velocity vectors were determined to be significantly amplified, particularly in the vicinity of the tall buildings. The velocity vectors were much longer than those in Case 1s, even in the even notch, because of the influence of the step-up and stepdown notches. In addition, this study observed phenomena in which the primary vortex center slanted toward the relatively low building, flow vectors separated at the top of the relatively tall building wall in the step-up notch, and wind on the pedestrian level tilted toward the upwind tall building. These results are similar to those reported by $\mathrm{Gu}$ et al. (2011), except for some slight differences engendered by secondary vortices. Velocity vectors observed for Case $3 \mathrm{~s}$ and $4 \mathrm{~s}$ were also noted to be similar to those obtained by $\mathrm{Gu}$ et al. (2011) and are not displayed herein.

\section{Effects of Time-varying Inflows}

This study compared the effects of time-varying and steady inflow conditions on the airflow pattern in each street canyon model. For Case 1, velocity vectors in the $x-z$ plane at $y=20 \mathrm{~m}$ and $t_{1}$ and $t_{2}$ (tagged in Fig. 6) are shown in Fig. 10. In contrast to those observed for Case 1s (Fig. 8(a)), the magnitudes of the velocity vectors in the street canyon were considerably enhanced. Under the condition of horizontal steady inflow, a strong horizontal shear layer formed near the roof layer. The shear layer drove the air inside the street canyon to move and form vortices. Zeng et al. (2010) demonstrated that gusty horizontal winds superimposed on basic wind flows would induce macroscopic vertical airflows with a downward velocity when the horizontal velocity is in the peak phase but with an upward velocity when the horizontal velocity is in the valley phase. The macroscopic vertical airflows would destroy the strong shear layer and directly exchange momentum with the air inside the street canyon (red streamlines in Fig. 10). At $t_{1}=350 \mathrm{~s}$, the horizontal velocity was determined to be in the peak phase, and a downward vertical airflow was induced and rushed into the street canyon from the vicinity of windward wall. At $t_{2}=1750 \mathrm{~s}$, the horizontal velocity was observed to be in the valley phase, and an upward vertical airflow was induced and caused the primary vortex to expand and gain momentum from the free stream.

To reveal the effect of the time-varying inflow condition on the airflow patterns in the uneven notches, Fig. 11 shows velocity vectors in the $x-z$ plane at $y=5 \mathrm{~m}$ (representing step-down notch) and $y=35 \mathrm{~m}$ (representing step-up notch) for Case 2. At $t_{1}=350 \mathrm{~s}$, the primary vortex disappeared in the step-up notch (Fig. 11(a)), and the inner air mass flowed out without restriction. However, the vortex vectors in the stepup notch were amplified (Fig. 11(c)) due to the enhancement of the free stream and downward vertical airflow induced by increasing horizontal inflow. At $t_{2}=1750 \mathrm{~s}$, the flow pattern in the step-down notch was rather complicated (Fig. 11(b)). The updraft induced by decreasing horizontal inflow suddenly sunk as it passed through the step-down notch, forming a vortex near the higher building that separated the existing primary vortex into two parts. The magnitude of the vortex vectors in the step-up notch deceased with the inflow velocity (Fig. 11(d)). In conclusion, the time-varying inflow condition could destroy the original flow structure in the step-down notch, and could influence the magnitude of the vortex vectors in the step-up notch. 
Composite Effects of Uneven Building Layouts and Timevarying Inflows

The vertical velocities near windward and leeward walls constitute a crucial variable for estimating the strength of

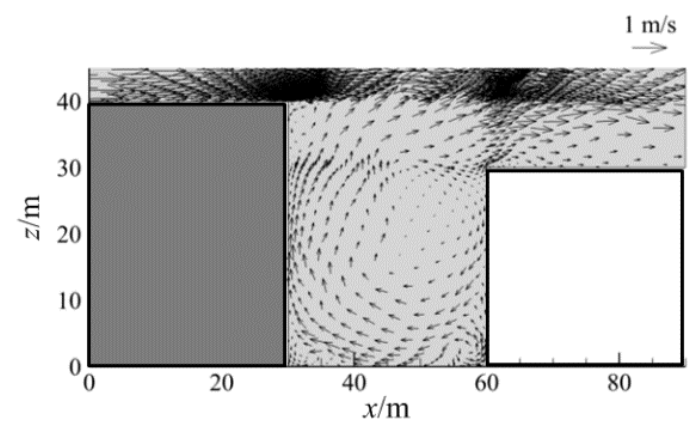

(a)

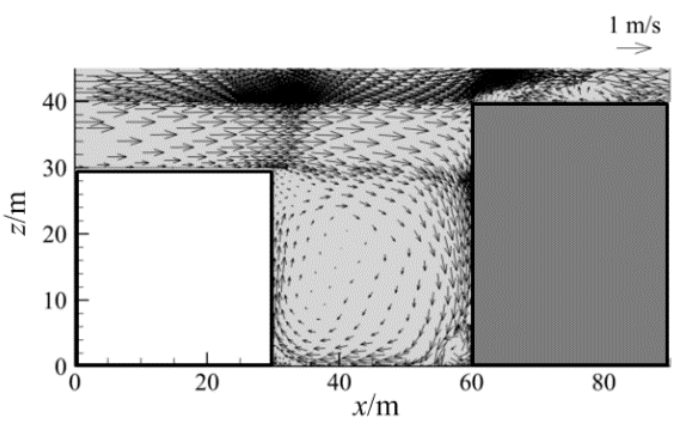

(c)

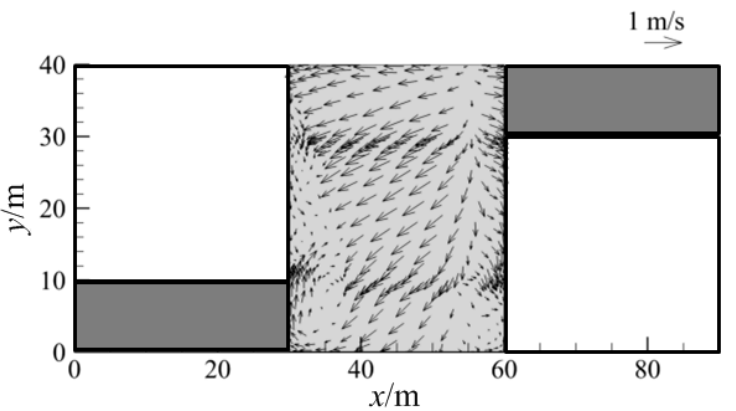

(f) airflow inside a street canyon. In this study, below a height of $40 \mathrm{~m}$ (the height of the relatively tall buildings in the models in the present study), the average vertical velocities were derived over a period of $1 \mathrm{~h}$ in the $y-z$ plane at $x=32 \mathrm{~m}$

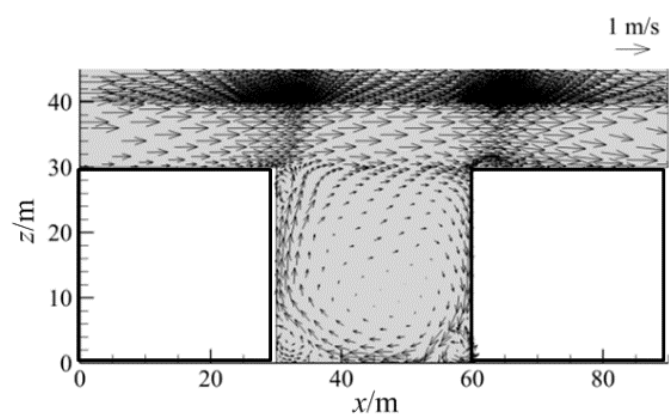

(b)

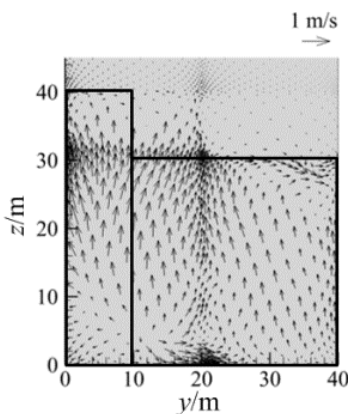

(d)

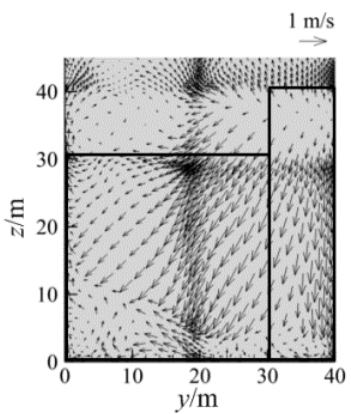

(e)

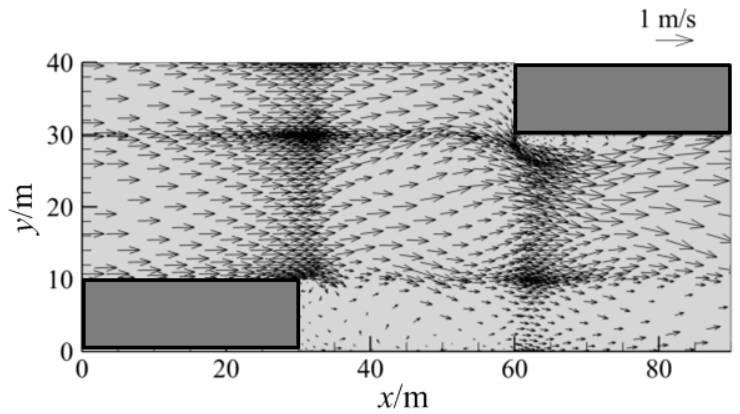

(g)

Fig. 9. Velocity vector distributions for Case $2 \mathrm{~s}$ in the (a) $x-z$ plane at $y=5 \mathrm{~m}$, (b) $x-z$ plane at $y=20 \mathrm{~m}$, (c) $x-z$ plane at $y$ $=35 \mathrm{~m}$, (d) $y-z$ plane at $x=32 \mathrm{~m}$ ( $2 \mathrm{~m}$ away from leeward wall), (e) $y-z$ plane at $x=58 \mathrm{~m}(2 \mathrm{~m}$ away from windward wall), (f) $x-y$ plane at $z=1.5 \mathrm{~m}$ (pedestrian level), (g) $x-y$ plane at $z=32 \mathrm{~m}$.

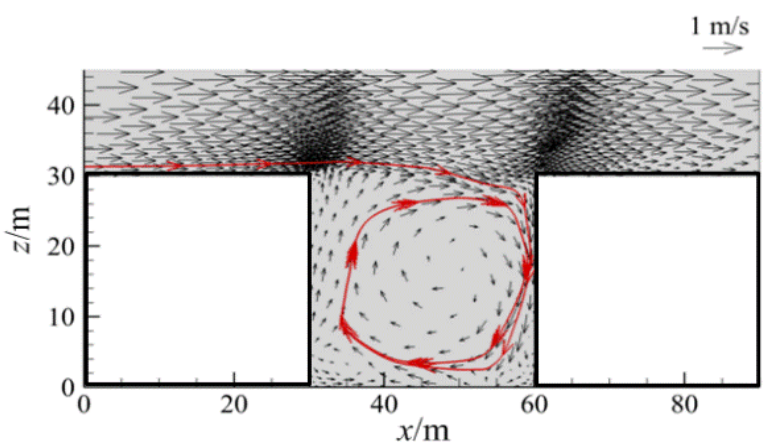

(a)

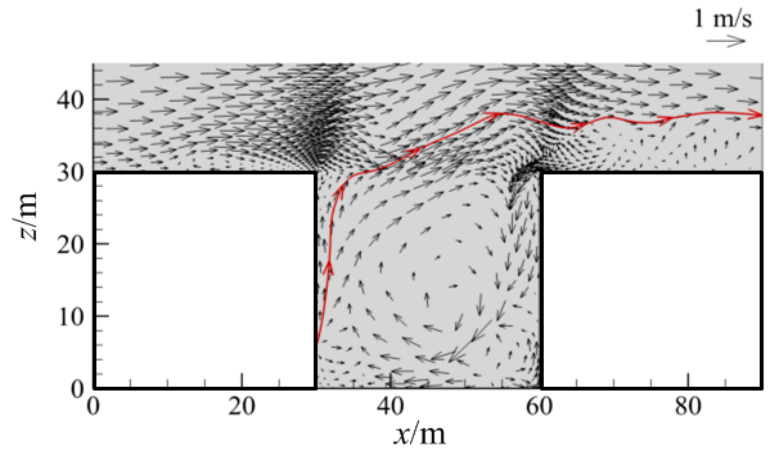

(b)

Fig. 10. Velocity vectors in the $x-z$ plane at $y=20 \mathrm{~m}$ at the moment of (a) $t_{1}=350 \mathrm{~s}$ and (b) $t_{2}=1750 \mathrm{~s}$. 


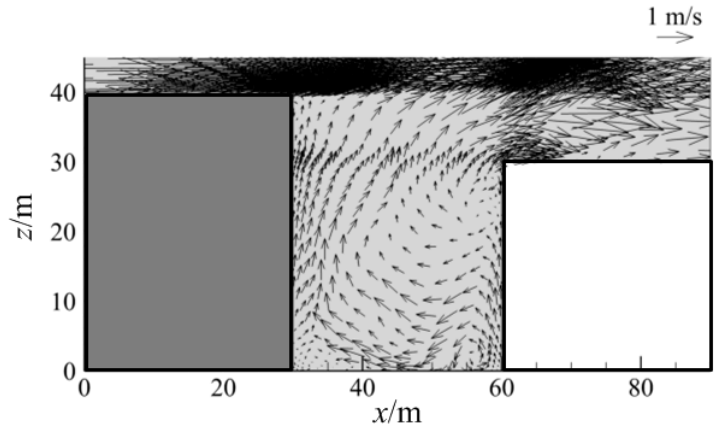

(a)

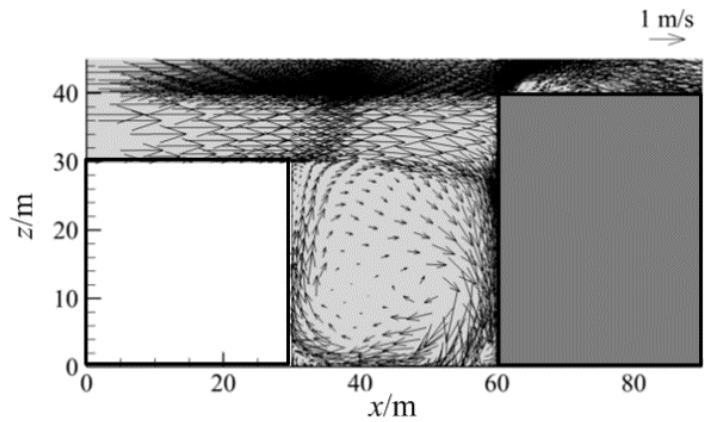

(c)

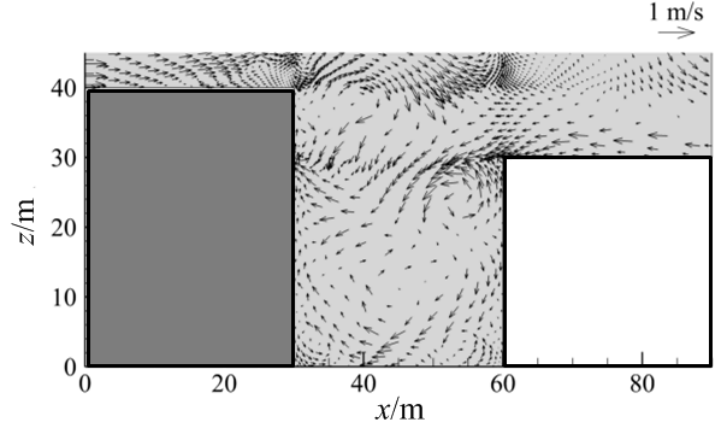

(b)

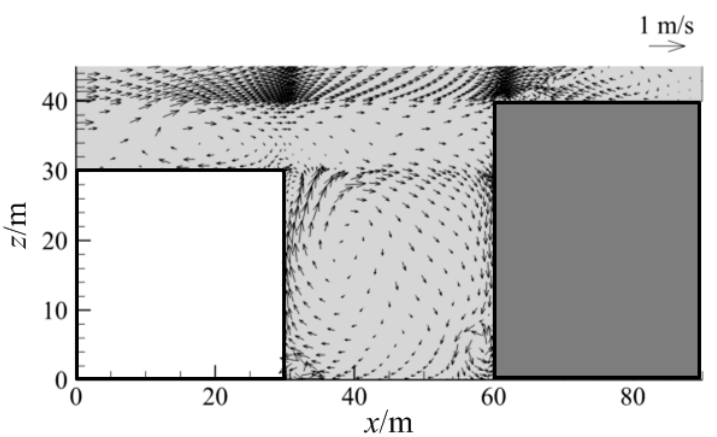

(d)

Fig. 11. Velocity vectors for Case 2 in the $x-z$ plane at $y=5 \mathrm{~m}$ at the moment of (a) $t_{1}=350 \mathrm{~s}$, (b) $t_{2}=1750 \mathrm{~s}$ and at $\mathrm{y}=35$ $\mathrm{m}$ at the moment of (c) $t_{1}=350 \mathrm{~s}$, (d) $t_{2}=1750 \mathrm{~s}$.

( $2 \mathrm{~m}$ away from leeward wall) and $x=58 \mathrm{~m}(2 \mathrm{~m}$ away from windward wall), as illustrated in Fig. 12. With consideration of the corresponding building layouts, the statistic plane was divided into three parts: step-down, even and step-up (Model 1 was determined to have only an even part, and Model 3 was determined to have no even part because of the adjoining of step-down and step-up notches). For Model 1, the time-space average vertical velocity driven by the timevarying inflow was amplified by approximately four times compared to that driven by the steady inflow; however, for any of the uneven models, this study observed no considerable enhancement of the strength of the airflow driven by the time-varying inflow inside the street canyon. The strength of the airflow inside the uneven street canyon models was obviously greater than that of the airflow inside the even model under the steady inflow condition, which would be difficult to maintain under time-varying inflow condition. In general, both an uneven building layout and time-varying inflow condition would enhance the strength of airflow inside a street canyon; nevertheless, the composite effects of these would not be the simple additive effects of uneven building layouts and time-varying inflow conditions on airflows. Furthermore, for all uneven models, the average vertical velocities in the vicinity of the taller buildings (step-down part at $x=32 \mathrm{~m}$ and step-up part at $x=58 \mathrm{~m}$ ) were always taller than those in the vicinity of the lower buildings (stepdown part at $x=58 \mathrm{~m}$ and step-up part at $x=32 \mathrm{~m}$ ) because of the wall effects of the taller buildings.

\section{Pollutant Distributions and Dispersions}

The pollutant distributions for all models under the steady inflow and time-varying inflow condition are presented in Figs. 13 and 14, respectively, in the form of the isoconcentration plane of a pollutant. The specified pollutant concentration was $0.05 \mu \mathrm{g} \mathrm{m}^{-3}$. Under the steady inflow condition, the pollutant transport direction was clockwise, and the transport was driven by the primary vortex; the pollutant accumulated inside the even street canyon model at the start time. Subsequently, some fraction of the pollutant was removed through the roof of the windward wall due to the action of turbulence (Figs. 13(a) and 13(e)). For the uneven street canyon models under the steady inflow condition, the pollutant was removed through only the roof of the relatively low building on the side of the windward wall because of the wall effect of the relatively tall building. Furthermore, the height of pollutant dispersion was maintained at nearly the height of the relatively tall building in the model under the steady inflow condition.

Under the time-varying inflow condition, the pattern of the pollutant isoconcentration plane also showed a time-varying characteristic. In addition, the height of pollutant dispersion was no longer restricted by the height of the relatively tall building when the inflow was in the valley phase; this was because of the induced updraft.

The mentioned pollutant isoconcentration planes reveal the pollutant distributions at only a certain time. To obtain the entire dispersion process over $1 \mathrm{~h}$, the average normalized pollutant concentration $C^{*}$ was calculated for all cases. The parameter $C^{*}$ can be defined as follows:

$$
C^{*}=\frac{V \cdot \bar{C}}{S_{c} \cdot V_{c} \cdot t}
$$




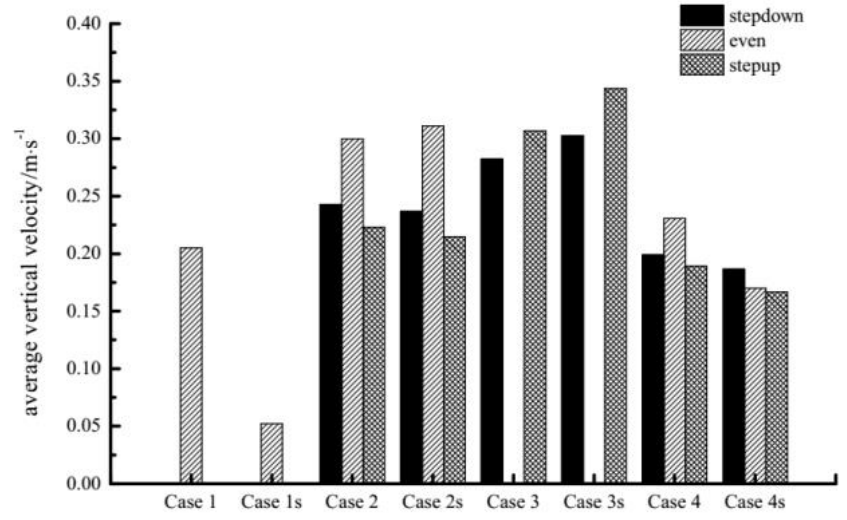

(a)

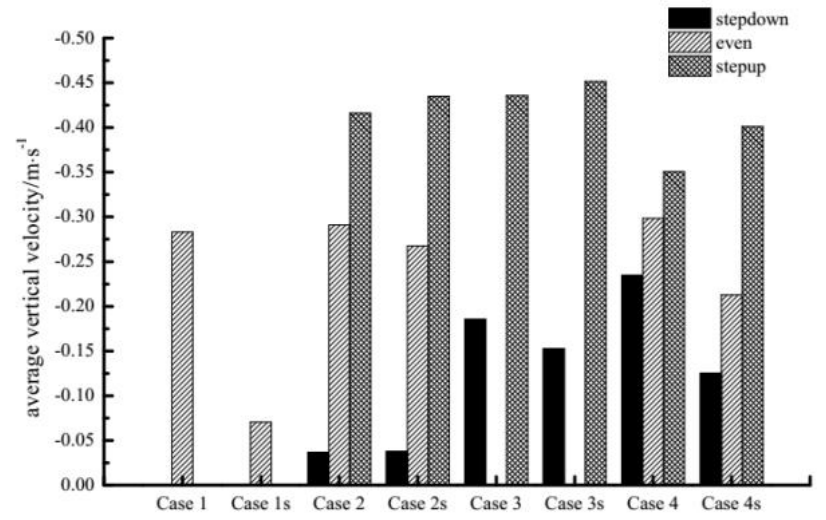

(b)

Fig. 12. Time-space average vertical velocity over 1 hour in the $y-z$ plane (below $z=40 \mathrm{~m}$ ) at (a) $x=32 \mathrm{~m}(2 \mathrm{~m}$ away from leeward wall) and (b) $x=58 \mathrm{~m}$ (2 $\mathrm{m}$ away from windward wall) for all cases.

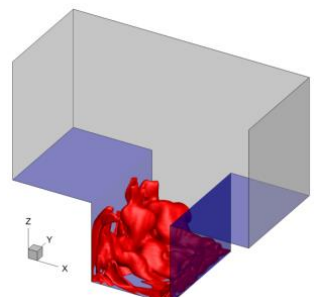

(a)

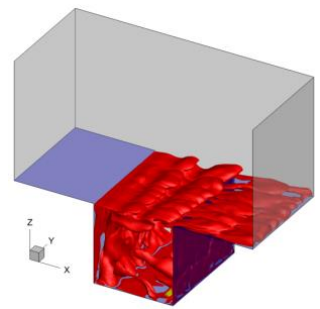

(e)

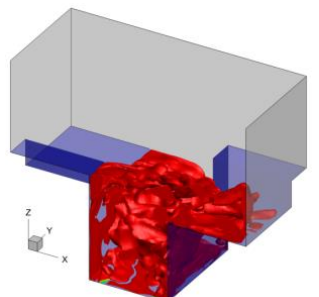

(b)

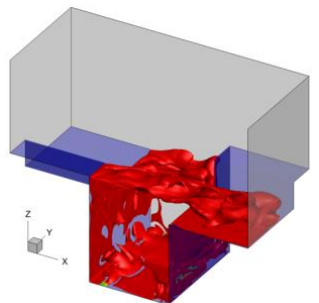

(f)

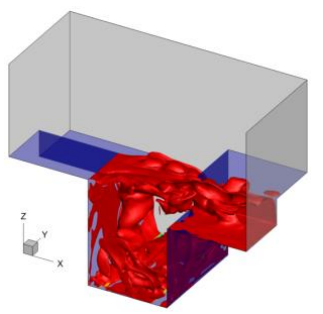

(c)

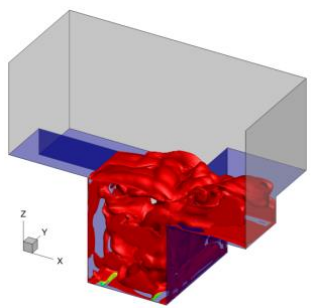

(g)

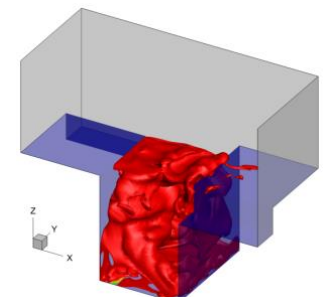

(d)

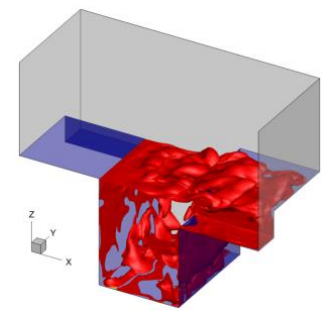

(h)

Fig. 13. Isoconcentration plane of a pollutant with a specified value of $0.05 \mu \mathrm{g} \mathrm{m}^{-3}$ at $t_{1}=350 \mathrm{~s}$ for (a) Case $1 \mathrm{~s}$, (b) Case $2 \mathrm{~s}$, (c) Case 3s, (d) Case $4 \mathrm{~s}$ and at $t_{2}=1750 \mathrm{~s}$ for (e) Case 1s, (f) Case 2s, (g) Case 3s, (h) Case 4s.

where $V$ is the volume of the calculated region, $\bar{C}$ is the timespace average pollutant concentration over a certain period, $S_{c}$ is the emission source intensity $\left(10 \mu \mathrm{g} \mathrm{m}^{-3} \mathrm{~s}^{-1}\right), V_{c}$ is the volume of the emission source, $t$ is the duration of pollutant emission. Notably, the initial value of $C_{*}$ in Fig. 15 represents the result obtained at $t=50 \mathrm{~s}\left(\left.C^{*}\right|_{t=0}=0\right)$. The average normalized pollutant concentration exhibited a smooth decreasing trend with a decreasing slope under the steady inflow condition, but the decreasing trend of $C *$ presents fluctuations under the time-varying inflow condition.

Fig. 16 presents a comparison of the time-space average normalized pollutant concentrations $\langle C *\rangle$ over a 1 -h period in all cases. The even street canyon model under the steady inflow condition (Case 1s) had the highest $\left\langle C_{*}\right\rangle$ value. Both the uneven building layouts and the time-varying inflows could improve pollutant dispersion inside the street canyon to some extent. The effect of the time-varying inflows on pollutant removal efficiency in the even building layout was determined to be more dramatic than that in the uneven building layouts. When the inflow condition was consistent, the best building layout for pollutant dispersion was determined to be Model 2, followed by Model 4, Model 3 and Model 1. The performance of the four building layouts with respect to pollutant dispersion under the steady inflow condition is supported by the results of Gu et al. (2011).

\section{CONCLUSIONS}

The airflow patterns and pollutant dispersion in four street canyon models, namely, one even model and three uneven models, under steady and time-varying inflow conditions were investigated in this study. The air flow in an even street canyon with a steady inflow is usually adopted as the benchmark in studies. Uneven building layouts are typically defined using the ORHB. The time-varying inflow condition was derived by averaging high-frequency velocity measurements over a reasonable amount of time that was estimated using a premultiplied power spectrum. Flow fields 


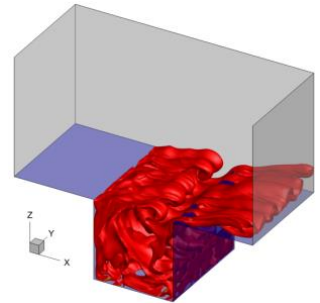

(a)

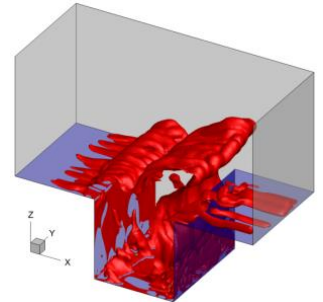

(e)

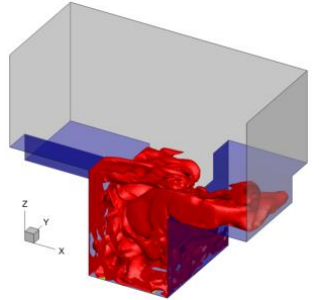

(b)

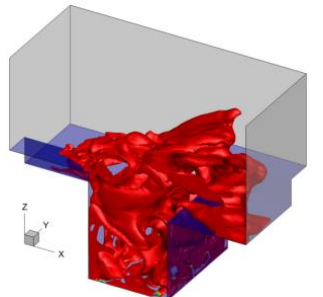

(f)

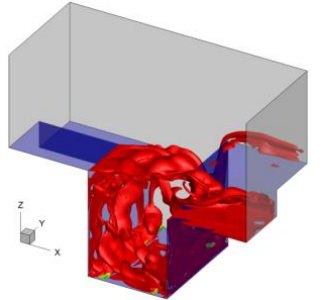

(c)

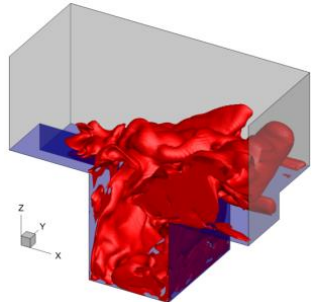

(g)

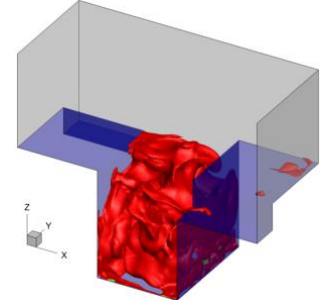

(d)

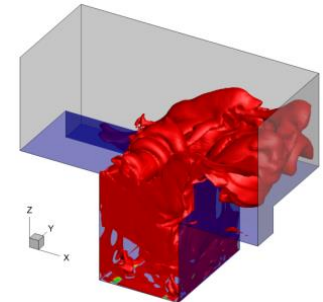

(h)

Fig. 14. Isoconcentration plane of pollutant concentration with a specified value of $0.05 \mu \mathrm{g} \mathrm{m}^{-3}$ at $t_{1}=350 \mathrm{~s}$ for (a) Case 1 , (b) Case 2, (c) Case 3, (d) Case 4 and at $t_{2}=1750 \mathrm{~s}$ for (e) Case 1, (f) Case 2, (g) Case 3, (h) Case 4.

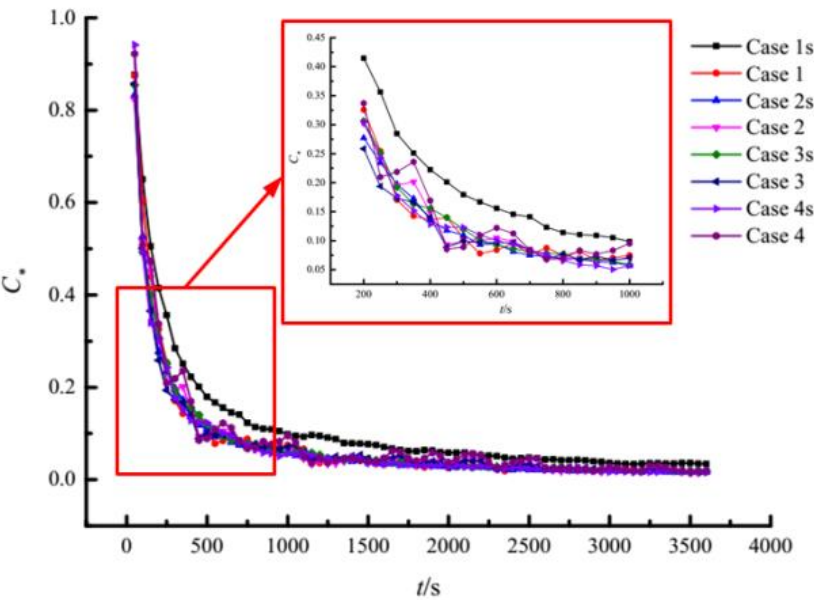

(a)

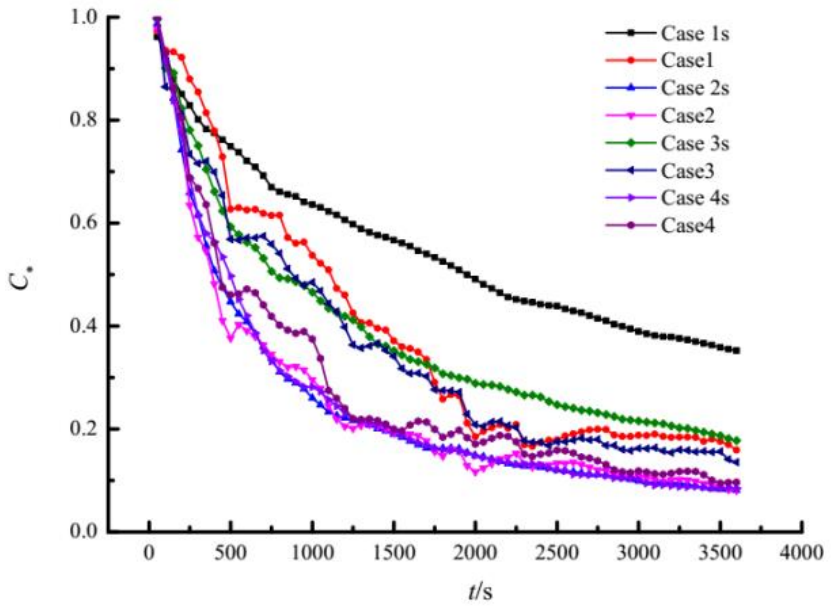

(b)

Fig. 15. Average normalized pollutant concentration (a) below $z=1.5 \mathrm{~m}$, (b) below $z=30 \mathrm{~m}$.

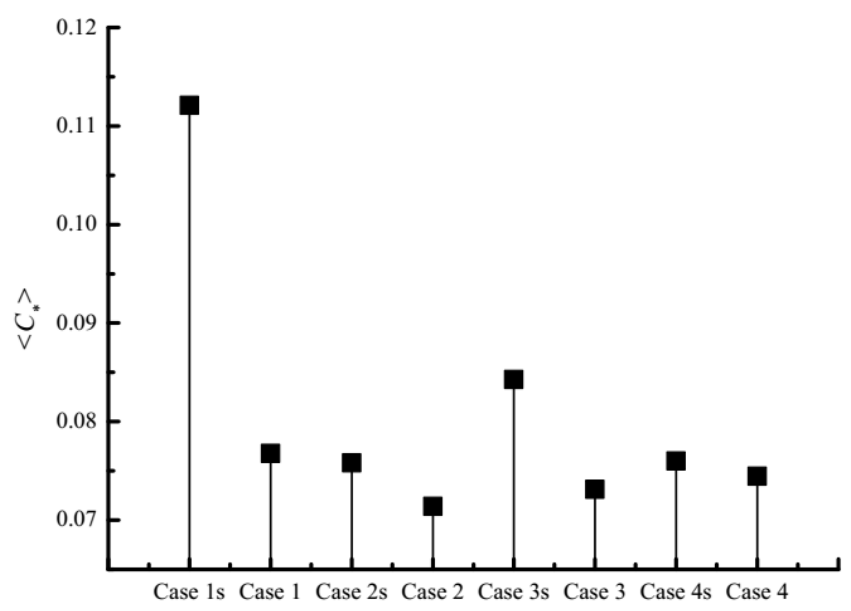

(a)

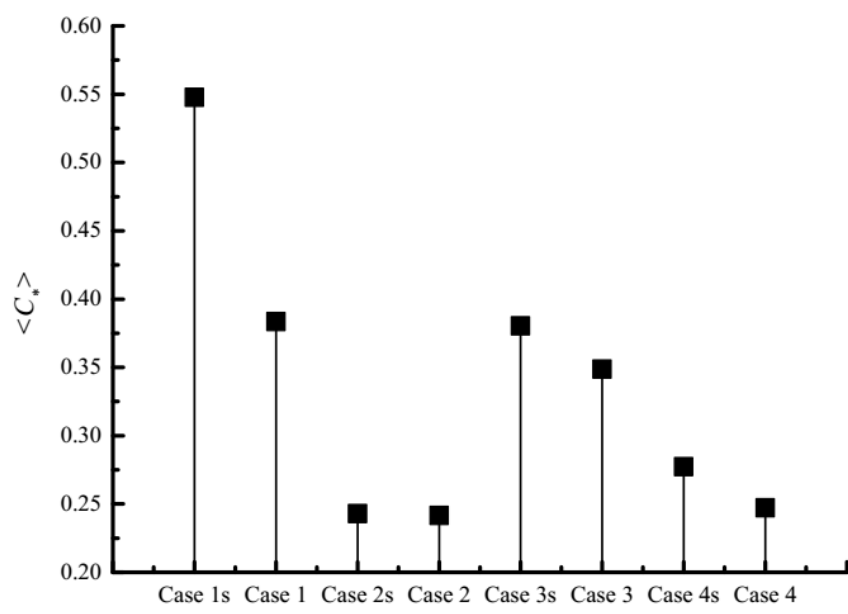

(b)

Fig. 16. The time-space average normalized pollutant concentrations $\left\langle C_{*}\right\rangle$ over 1 hour (a) below $z=1.5 \mathrm{~m}$, (b) below $z=$ $30 \mathrm{~m}$. 
in the different street canyon models under the steady inflow condition were solved using the PISO algorithm, whereas those under the time-varying inflow condition were solved using a new scheme based on the standard SIMPLE algorithm formulated on the OpenFOAM platform.

With a steady inflow, the velocity vectors were significantly amplified because of the uneven building layouts, particularly in the vicinity of high-rise buildings. With the time-varying inflow, macroscopic vertical air flows occurred above the roof layer in all of the models; these air flows destroyed the strong shear layer and directly exchanged momentum with the air inside the street canyon. In all of the cases, a comparison between the average vertical velocity near the windward wall and that near the leeward wall showed that both an uneven building layout and a time-varying inflow condition enhanced the air flow-and thus the pollutant removal efficiency - inside the street canyon. However, the composite effects of these two factors are not simply additive.

In the uneven street canyons with the steady inflow, the dispersion of pollutants occurred through the roofs of the low-rise buildings to the side of the windward wall and extended vertically nearly to the layer of roofs for the highrise buildings. However, with the time-varying inflow, the dispersion was no longer restricted by the high-rise buildings due to the induced updrafts during inflow in the valley phase. The pollutant isoconcentration plane also exhibited temporal variation, as illustrated by the fluctuant time-series curves of the normalized concentrations. Furthermore, the average time-space normalized pollutant concentrations $\left\langle C_{*}\right\rangle$ revealed that Case $1 \mathrm{~s}$, the even street canyon with the steady inflow, exhibited the poorest performance with respect to dispersion.

This study applied LES to investigate the composite effects of an uneven building layout and a time-varying inflow on the air flow pattern and the pollutant dispersion in a street canyon. In the future. This study investigated the, the influence of additional factors, such as wall heating and variation in the inflow direction, will be studied, and the simulation results will be validated through scale-model outdoor field measurement.

\section{ACKNOWLEDGEMENTS}

This work was supported by the National Natural Science Foundation of China (No. 11572242, 41861144021) and the Shaanxi Key Development Project of Industrial Innovation Chain (No. 2018ZDCXL-SF-02-06). This work was also supported by the National Key R\&D Program of China (No. 2016ZX05011003). This manuscript was edited by Wallace Academic Editing.

\section{SUPPLEMENTARY MATERIAL}

Supplementary data associated with this article can be found in the online version at http://www.aaqr.org.

\section{REFERENCES}

Ahmad, K., Khare, M. and Chaudhry, K.K. (2005). Wind tunnel simulation studies on dispersion at urban street canyons and intersections-A review. J. Wind Eng. Ind. Aerodyn 93: 697-717. https://doi.org/10.1016/j.jweia.2005. 04.002

Bady, M., Kato, S. and Huang, H. (2008). Towards the application of indoor ventilation efficiency indices to evaluate the air quality of urban areas. Build. Environ. 43: 1991-2004. https://doi.org/10.1016/j.buildenv.2007.11.013

Baik, J.J. and Kim, J.J. (1999). A numerical study of flow and pollutant dispersion characteristics in urban street canyons. J. Appl. Meteorol. 38: 1576-1589. https://doi.org/ 10.1175/1520-0450(1999)038<1576:ANSOFA > 2.0.CO;2

Baik, J.J., Park, R.S., Chun, H.Y. and Kim, J.J. (2000) A laboratory model of urban street-canyon flows. J. Appl. Meteorol. 39: 1592-1600. https://doi.org/10.1175/15200450(2000)039<1592:ALMOUS>2.0.CO;2

Chan, C.K. and Yao, X. (2008). Air pollutant in mega cities in China. Atmos. Environ. 42: 1-42. https://doi.org/10.1016 /j.atmosenv.2007.09.003

Duan, G. and Naga, K. (2018). Effects of time-dependent inflow perturbations on turbulent flow in a street canyon. Boundary Layer Meteorol. 167: 257-284. https://doi.org/ 10.1007/s10546-017-0327-1

Eliasson, I., Offerle, B., Grimmond, C.S.B. and Lindqvist, S. (2006) Wind fields and turbulence statistics in an urban street canyon. Atmos. Environ. 40: 1-16. https://doi.org/ 10.1016/j.atmosenv.2005.03.031

Fazia, A.T. and Helmut, M. (2006). Numerical study on the effects of aspect ratio and orientation of an urban street canyon on outdoor thermal comfort in hot and dry climate. Build. Environ. 41: 94-108. https://doi.org/10.1016/j.build env.2005.01.013

Gu, Z.L., Zhang, Y.W., Cheng, Y. and Lee, S.C. (2011). Effect of uneven building layout on air flow and pollutant dispersion in non-uniform street canyons. Build. Environ. 46: 2657-2665. https://doi.org/10.1016/j.buildenv.2011. 06.028

Hang, J., Li, Y., Sandberg, M., Buccolieri, R. and Di Sabatino, S. (2012). The influence of building height variability on pollutant dispersion and pedestrian ventilation in idealized high-rise urban areas. Build. Environ. 56: 346-360. https://doi.org/10.1016/j.buildenv.2012.03.023

Hang, J., Sandberg, M., Li, Y. and Claesson, L. (2009). Pollutant dispersion in idealized city models with different urban morphologies. Atmos. Environ. 43: 60116025. https://doi.org/10.1016/j.atmosenv.2009.08.029

Hutchins, N., Chauhan, K., Marusic, I., Monty, J. and Klemicki, J. (2012). Towards reconciling the large-scale structure of turbulent boundary layers in the atmosphere and laboratory. Boundary Layer Meteorol. 145: 273-306. https://doi.org/10.1007/s10546-012-9735-4

Issa, R.I. (1986). Solution of the implicit discretized fluid flow equations by operator splitting. J. Comput. Phys. 62: 40-65. https://doi.org/10.1016/0021-9991(86)90099-9

Kim, J.J. and Baik, J.J. (2004). A numerical study of the effects of ambient wind direction on flow and dispersion in urban street canyons using the RNG $\mathrm{k}-\varepsilon$ turbulence model. Atmos. Environ. 38: 3039-3048. https://doi.org/10. 1016/j.atmosenv.2004.02.047

Kim, K.C. and Adrian, R.J. (1998). Very large-scale motion 
in the outer layer. Phys. Fluids 11: 417-422. https://doi.org/ $10.1063 / 1.869889$

Krecl, P., Targino, A. C., Johansson, S. and Ström, J. (2015). Characterisation and source apportionment of submicron particle number size distributions in a busy street canyon. Aerosol Air Qual. Res. 15: 220-233. https://doi.org/10.4 209/aaqr.2014.06.0108

Kumar, P., Garmory, A., Ketzel, M., Berkowicz, R. and Britter, R. (2009). Comparative study of measured and modelled number concentrations of nanoparticles in an urban street canyon. Atmos. Environ. 43: 949-958. https://doi.org/10.1016/j.atmosenv.2008.10.025

Li, W.J., He, Y.P., Zhang, Y.W., Su, J.W., Chen, C.G, Yu, C.W., Zhang, R.J. and Gu, Z.L. (2019a). LES simulation of flow field and pollutant dispersion in a street canyon under time-varying inflows with TimeVarying-SIMPLE approach. Build. Environ. 157: 185-196. https://doi.org/ 10.1016/j.buildenv.2019.04.049

Li, W.J., Zhang, Y., Yang, B., Su, J.W., Zhang, Y.W., Lu, W.Z., Shui, Q.X., Wu, X.Y., He, Y.P. and Gu, Z.L. (2019b). Large-scale turbulence structures in a laboratoryscale boundary layer under steady and gusty wind inflows. Sci. Rep. 9: 9397. https://doi.org/10.1038/s41598019-45873-X

Li, X., Liu, C.H., Leung, D.Y.C. and Lam, K.M. (2006). Recent progress in CFD modelling of wind field and pollutant transport in street canyons. Atmos. Environ. 40: 5640-5658. https://doi.org/10.1016/j.atmosenv.2006.04.055

Li, X.L., Wang, J.S., Tu, X.D., Liu, W. and Huang, Z. (2007). Vertical variations of particle number concentration and size distribution in a street canyon in Shanghai, China. Sci. Total Environ. 378: 306-316. https://doi.org/10.1016/j.scitotenv.2007.02.040

Li, X.X., Liu, C.H. and Leung, D.Y.C. (2008). Large-eddy simulation for flow and pollutant dispersion in highaspect-ratio urban street canyons with wall model. Boundary Layer Meteorol. 129: 249-268. https://doi.org/ 10.1007/s10546-008-9313-y

Li, X.X., Liu, C.H. and Leung, D.Y.C. (2009). Numerical investigation of pollutant transport characteristics inside deep urban street canyons. Atmos. Environ. 43: 24102418. https://doi.org/10.1016/j.atmosenv.2009.02.022

Lin, L., Hang, J., Wang, X.X., Wang, X. M., Fan, S.J., Fan, Q. and Liu, Y.H. (2016). Integrated effects of street layouts and wall heating on vehicular pollutant dispersion and their reentry into downstream canyons. Aerosol Air Qual. Res. 16: 3142-3163. https://doi.org/10.4209/aaqr. 2016.04.0148

Liu, F.Q. (2016). A thorough description of how wall functions are implemented in Open FOAM. Proceedings of CFD with OpenSource Software, 2016, Nilsson, H. (Ed.), http://www.tfd.chalmers.se/ hani/kurser/OS_CFD_2016 /FangqingLiu/openfoamFinal.pdf

Liu, S., Miao, G., Zhang, C.L., Cui, G.X. and Zhang, Z.S. (2012). Study on micro-atmospheric environment by coupling large eddy simulation with mesoscale model. $J$. Wind Ind. Aerod. 107-108: 106-117. https://doi.org/10.10 16/j.jweia.2012.03.033

Nelson, M.A., Pardyjak, E.R., Klewicki, J.C., Pol, S.U. and
Brown, M.J. (2007). Properties of the wind field within the Oklahoma City Park Avenue street Canyon. Part I: mean flow and turbulence statistics. J. Appl. Meteorol. Clim. 46: 2038-2054. https://doi.org/10.1175/2006JAM C1427.1

Oke, T.R. (1998). Street design and urban canopy layer climate. Energy Build. 11: 103-113. https://doi.org/10.1016 /0378-7788(88)90026-6

Patankar, S.V. and Spalding, D.B. (1972). A calculation procedure for heat, mass and momentum transfer in threedimensional parabolic flows. Int. J. Heat Mass Transfer 15: 1787-1806. https://doi.org/10.1016/0017-9310(72)90 054-3

Pope, S.B. (2000). Turbulent flows. Cambridge University Press, Cambridge, UK.

Sabatino, S.D., Buccolieri, R., Pulvirenti, B. and Britter, R. (2007). Simulations of pollutant dispersion within idealised urban-type geometries with CFD and integral models. Atmos. Environ. 41: 8316-8329. https://doi.org/ 10.1016/j.atmosenv.2007.06.052

Sagrado, A.P.G., Beeck, J., Rambaud, P. and Olivari, D. (2002). Numerical and experimental modelling of pollutant dispersion in a street canyon. J. Wind Eng. Ind. Aerod. 90: 321-339. https://doi.org/10.1016/S0167-6105(01)00215-X

Scungio, M., Arpino, F., Stabile, L. and Buonanno, G. (2013). Numerical simulation of ultrafine particle dispersion in urban street canyons with the spalartallmaras turbulence model. Aerosol Air Qual. Res. 13: 1423-1437. https://doi.org/10.4209/aaqr.2012.11.0306

Smagorinsky, J. (1963). General circulation experiments with the primitive equations: I. The basic experiment. Mon. Weather Rev. 91: 99-164. https://doi.org/10.1175/ 1520-0493(1963)091<0099:GCEWTP>2.3.CO;2

Smits, A.J., McKeon, B.J. and Marusic, I. (2011). HighReynolds number wall turbulence. Annu. Rev. Fluid Mech. 43: 53-75. https://doi.org/10.1146/annurev-fluid122109-160753

Su, J.W., Chai, G.L., Wang, L., Cao, W.D., Gu, Z.L., Chen, C.G. and Xu, X.Y. (2019). Pore-scale direct numerical simulation of particle transport in porous media. Chem. Eng. Sci. 199: 613-627. https://doi.org/10.1016/j.ces.2019. 01.033

Tominaga, Y. and Stathopoulos, T. (2011). CFD modeling of pollution dispersion in a street canyon: Comparison between LES and RANS. J. Wind Eng. Ind. Aerod. 99: 340-348. https://doi.org/10.1016/j.jweia.2010.12.005

Vardoulakis, S., Fisher, B.E.A., Pericleous, K. and GonzalezFlesca, N. (2003). Modelling air quality in street canyons: A review. Atmos. Environ. 37: 155-182. https://doi.org/ 10.1016/S1352-2310(02)00857-9

Wang, G.H. and Zheng, X.J. (2016). Very large scale motions in the atmospheric surface layer: A field investigation. J. Fluid. Mech. 802: 464-489. https://doi.org/ 10.1017/jfm.2016.439

Wang, Q., Wang, Y., Zhao, J.Y. and Bai, C.X. (2015). Diffusion factors of street canyon pollutants in the cold winter of Xi'an city based on back propagation neural network analysis. Indoor Built. Environ. 24: 1082-1094. https://doi.org/10.1177/1420326X15583918 
Welch, P. (1967). The use of fast Fourier transform for the estimation of power spectra: A method based on time averaging over short, modified periodograms. IEEE Trans. Audio Electroacoust. 15: 70-73.

Xie, X.X., Huang, Z. and Wang, J.S. (2006). The impact of urban street layout on local atmospheric environment. Build. Environ. 41: 1352-1363. https://doi.org/10.1016/ j.buildenv.2005.05.028

Yim, S.H.L., Fung, J.C.H., Lau, A.K.H. and Kot, S.C. (2009). Air ventilation impacts of the "wall effect" resulting from the alignment of high-rise buildings. Atmos. Environ. 43: 4982-4994. https://doi.org/10.1016/ j.atmosenv.2009.07.002

Zeng, Q.C., Cheng, X.L. and Hu, F. (2007). The mechanism of soil erosion and dust emission under the action of nonsteady strong wind with descending motion and gustwind Clim. Environ. Res. 12: 244-250. (in Chinese)

Zeng, Q.C., Cheng, X.L., Hu, F. and Peng, Z. (2010). Gustiness and coherent structure of strong winds and their role in dust emission and entrainment. Adv. Atmos. Sci. 27: 1. https://doi.org/10.1007/s00376-009-8207-3

Zhang, K., Chen, G.W., Wang, X.M., Liu, S.H., Mak, C.M., Fan, Y.F. and Hang, J. (2019). Numerical evaluations of urban design technique to reduce vehicular personal intake fraction in deep street canyons. Sci. Total Environ. 653: 968-994. https://doi.org/10.1016/j.scitotenv.2018.10.333

Zhang, Y.W., Gu, Z.L., Chen, Y. and Lee, S.C. (2011a). Effect of real-time boundary wind conditions on the air flow and pollutant dispersion in an urban street canyonLarge eddy simulations. Atmos. Environ. 45: 3352-3359. https://doi.org/10.1016/j.atmosenv.2011.03.055

Zhang, Y.W., Gu, Z.L., Lee, S.C., Fu, T.M. and Ho, K.F. (2011b). Numerical simulation and in Situ investigation of fine particle dispersion in an actual deep street canyon in Hong Kong. Indoor Built. Environ. 20: 206-216. https://doi.org/10.1177/1420326X10387694

Zhang, Y.W., Gu, Z.L. and Yu, C.W. (2012). Time-Series numerical simulation of wind flow within urban canopy layer and its integration effect for prediction of pollutant concentration variation. Indoor Built. Environ. 21: 355357. https://doi.org/10.1177/1420326X12441698

Zhang, Y.W., Gu, Z.L., Wang, Z.S. and Yu, C.W. (2013). Revisit of wind flows between wind tunnels and real canyons-the viewpoint of Reynolds Dynamic Similarity. Indoor Built. Environ. 22: 467-470. https://doi.org/10.1177/ 1420326X12489064

Zhang, Y.W., Gu, Z.L. and Yu, C.W. (2018). Review on numerical simulation of airflow and pollutant dispersion in urban street canyons under natural background wind condition. Aerosol Air Qual. Res. 18: 780-789. https://doi.org/10.4209/aaqr.2017.09.0303

Received for review, June 26, 2019

Revised, November 29, 2019 Accepted, March 9, 2020 